\title{
Relationship between Cleavage Rate and Porcine Semen Fertility
}

\author{
Julian Valencia, Juan Marín and Francisco Javier Henao* \\ Doctoral Program in Agrarian Sciences, University of Caldas, Manizales, Colombia
}

Submission: August 16, 2018; Published: September 11, 2018

*Corresponding author: Francisco Javier Henao, Doctoral Program in Agrarian Sciences, University of Caldas, Manizales, Colombia, Email: fhenao@ucaldas.edu.co

\begin{abstract}
The aim of the present investigation was to establish the value of the rate of cleaved zygotes (RCZ) as an in vitro reference test to evaluate the fertilizing capacity in commercial semen samples for those cases in which it is impossible to perform a field fertility evaluation with an enough number of sows under strictly controlled conditions. Fifteen boars were ejaculated every 7 days in three consecutive times, and the sperm-rich fraction was diluted and cooled to 17 to perform seminal analysis. Friedman test and Spearman correlation were performed. Boars were grouped according to two groups of RCZ ( $<23 \%$ and $>23 \%$ ) and Kruskal-Wallis test was performed to detect differences between groups. All boars averaging $<23 \%$ of RCZ had low seminal quality and all boars with an average above this value had high seminal quality. There was no significative difference in RCZ within boars. Progressive motility (PM), total motility (TM) and normal morphology (NM) were different between two RCZ groups. A high and significative correlation of RCZ with PM and NM was observed. The results found in this research allow to establish the RCZ (threshold of 23\%) as an in vitro reference test because its consistence and repeatability to evaluate fertilizing capacity in commercial semen samples.
\end{abstract}

Keywords: Cleaved zygotes; In vitro Fertilization; Seminal Quality; Boar Semen; Boar Fertility

Abbreviations: RCZ: Rate of Cleaved Zygotes; TM: Total Motility; PM: Progressive Motility; NM: Normal Morphology; MSI: Membrane Structural Integrity; MFI: Membrane Functional Integrity; sHOST: Short Hypoosmotic Swelling Test; CASA: Computer Assisted Semen Analysis; AI: Acrosomal Integrity; IVF: In Vitro Fertilization; ZP: Zona Pellucida; IVM: In Vitro Maturation; mDPBS: Modified Dulbecco's PhosphateBuffered Saline; BSA: Bovine Serum Albumin; IVC: In Vitro Culture

\section{Introduction}

In boars, the production of fertile spermatozoa is considered an essential condition by which it is evaluated their reproductive merit. Research in boar fertility has been oriented mainly toward the qualitative and quantitative characteristics of semen. Semen quality or fertility is the ability of semen to generate live pigs; and quantity, understood as the number of spermatozoa produced [1].

Evaluation of qualitative semen characteristics, as: morphology, concentration, membrane structural integrity (MSI), and subjective assessment of motility (PM and TM), is routinely carry out in artificial insemination centers. PM, TM and MSI have been used to eliminate semen samples with very low seminal quality, but their relationship with fertility is unclear [2]. Due to these variables do not permit to predict relative fertility in boars with good quality semen [3], it is imperative to perform field tests to associate the in vitro analysis with fertility and prolificacy, using a statistically enough sows under controlled conditions. Carry out such evaluations under the real conditions of commercial pig farms is practically utopian, and it is only possible in large centres dedicated exclusively to research.

To try to solve this problem, higher resolution methods have been developed to evaluate different seminal characteristics such as: MSI through staining with SYBR-14 and propidium iodide [4], membrane functional integrity (MFI) by the short hypoosmotic swelling test (sHOST) [5], and the objective evaluation of sperm motility (Computer Assisted Semen Analysis, CASA). There is a moderate correlation between MFI and farrowing rate [5], which could suggest that MFI is an important characteristic in the fertilization process [6]. In relation with MSI and objective evaluation of motility, these methods really provide more information on the integrity and functionality of the sperm cells [7], than on the necessary characteristics in fertilization [3]. The in vitro fertilization (IVF) systems are recognized as very good tools to evaluated semen quality [8]. These systems provide relevant information about sperm capacitation, acrosome reaction, oocyte fertilization and embryonic development [9]. The zona-free hamster oocytes penetration test has been one 
of the first systems employed in this aim, with the obvious limitations about recognition, union and penetration of the zona pellucida (ZP) due to the used oocytes [10]; problems overcome with the use of oocytes from prepubertal gilts in penetration tests and ZP-binding assays [8].

Thanks to greater associated polyspermia, immature oocytes in germinal vesicle stage have been considered for evaluation of sperm penetration rate and average number of spermatozoa per oocyte, which are variables that permit discriminate boars with high, intermediate or low fertility [2].Through the use of in vitro maturated oocytes, it is possible to evaluate gamete recognition, penetration and pronucleus formation [3]. If furthermore the fertilized oocytes are cultured in vitro, the RCZ and of blastocyst formation can be evaluated [11]. Rate of cleaved zygotes and rate of blastocyst formation have been considerate the best estimators of semen fertility [8].

The aim of the present investigation was to establish the value of the RCZ as an in vitro reference test to evaluate the fertilizing capacity in commercial semen samples for those cases in which it is impossible to perform a field fertility evaluation with an enough number of sows under strictly controlled conditions.

\section{Materialand methods}

\section{Animals}

Fifteen boars between 15 and 21-month-old of two insemination centers in the central-western of Colombia (nine boars from CERCAFE and 6 boars from Topigenética), with normal semen analysis, optimal sanitary and fertility conditions were used for the study. They were kept in individual $2.6 \times 2.5 \mathrm{~m}$ pens, fed $2 \mathrm{~kg} / \mathrm{animal} / \mathrm{d}$ of a commercial feed for boars, water ad libitum, and ejaculated every 7 days between 07:00 and 09:00h.

\section{Semen Collection and Processing}

The fifteen boars were ejaculated three consecutive times (45 ejaculates in total) using the gloved hand technique. The rich fraction of each male was separated visually, diluted 1:1.5 with Androhep® plus (Minitüb, Germany), cooled to $17^{\circ} \mathrm{C}$ in three hours and transported to the Laboratory of Biology Reproduction at University of Caldas where it was rediluted to 30 x 106 spermatozoa/mL to initiate the seminal quality analysis.

\section{Evaluationof Sperm Variables}

Sperm Motility: It was evaluated by CASA system and software Sperm Class Analyzer (Ver. 6.2, 2015; Microptic S.L., Barcelona, Spain). In this, a $50 \mu \mathrm{L}$ aliquot of semen was diluted at $15 \times 106$ spermatozoa/mL in BTS and incubated for 5 min in a water-bath at $37{ }^{\circ} \mathrm{C}$. Four fields and a minimum of 400 spermatozoa were analysed.

Membrane Structural Integrity: It was developed by staining with SYBR-14 and propidium iodide (LIVE/ DEAD®Sperm Viability Kit L-7011, Molecular Probes, Europe), incubation for $5 \mathrm{~min}$ at 37 in a water bath, and visualization of
100 spermatozoa at 1,000 $\times$ magnification in anepifluorescence microscope (Nikon Eclipse 80i, Japan) with BV-2A filter [4].

Membrane Functional Integrity: It was evaluated through the sHOST, and examination of 100 spermatozoa at $400 \times$ magnification in a phase-contrast microscope (Nikon Eclipse 50i, Japan) after exposure to a hypoosmotic solution of $75 \mathrm{mOsm} / \mathrm{kg}$ during $5 \mathrm{~min}$ at 37 [?5].

Acrosomal integrity (AI): AI was analyzed in sperm fixed with $2 \%$ glutaraldehyde, and examination of apical ridge in 100 spermatozoa at $1,000 \times$ magnification in a phase-contrast microscope according to the criteria of Pursel and Johnson [12].

Sperm Morphology: of head, middle piece and tail, cytoplasmic droplet and agglutination were development by counting 100 spermatozoa in semen fixed with formalin saline solution, at $400 \times$ magnification in a phase contrast microscope.

\section{In Vitro Fertilization and Culture}

The IVF, in vitro maturation (IVM) and in vitro culture (IVC) were performed as described by Abeydeera and Day [13].

CultureMedia: Modified Dulbecco's phosphate-buffered saline (mDPBS) medium, which is DPBS supplemented with $4 \mathrm{mg} / \mathrm{mL}$ of bovine serum albumin (BSA) (fraction V, SIGMA, A7888, initial fractionation by cold alcohol precipitation), $0.34 \mathrm{mM}$ sodium pyruvate, $5.4 \mathrm{mM} \mathrm{D}$-glucose and $70 \mathrm{mg} / \mathrm{mL}$ of kanamycin was used for the collection, washing and selection of Cumulus-oocyte-complexes (COC's). The medium NCSU-23 BSA-free supplemented with $10 \%$ (v:v) porcine follicular fluid, $10 \mathrm{ng} / \mathrm{mL}$ epidermal growth factor, $0.1 \mathrm{mg} / \mathrm{mL}$ cysteine, $10 \mathrm{IU} /$ mL eCG (Sigma, G4877), and 10 IU/mL hCG (Sigma, C1063), was used for the IVM of oocytes. Modified Tris-buffered medium supplemented with $2 \mathrm{mM}$ caffeine and $0.2 \%$ BSA (fraction V, A7888 Sigma) was used for IVF. NCSU-23 with $0.4 \%$ BSA (fraction V, A7888 Sigma) was used for embryo culture. Porcine follicular fluid was collected from follicles of 3-6mm in diameter, centrifuged at $1900 \times \mathrm{g}$ for $30 \mathrm{~min}$ at 4 ? , filtered through $1.2 \mu \mathrm{m}$ and $0.22 \mu \mathrm{m}$ syringes filters, and stored at -20 until use.

Oocyte Collection and in Vitro Maturation: Ovaries were collected from prepubertal gilts at a local slaughterhouse and transported immediately to the laboratory, between 30-34 ?, in $0.9 \%(\mathrm{w} / \mathrm{v}) \mathrm{NaCl}$ with kanamycin. COC's were aspirated from follicles with $3-6 \mathrm{~mm}$ of diameter, using a $10 \mathrm{~mL}$ syringe and an 18-gauge needle. COC's with several compact layers of cumulus cells and uniform dark cytoplasm were selected. Groups of 50 COC's were matured in $500 \mu \mathrm{L}$ of IVM medium with hormones for $22 \mathrm{~h}$ at $5 \%$ CO2 and 39 ? and in $500 \mu \mathrm{L}$ of IVM medium without hormones for $22 \mathrm{~h}$ more.

Semen preparation: Twenty $\mathrm{mL}$ of semen diluted and refrigerated for $24 \mathrm{~h}$ at 17 were centrifuged at $100 \times \mathrm{g}$ for $3 \mathrm{~min}$, and the supernatants were centrifuged at $1200 \times \mathrm{g}$ for $3 \mathrm{~min}$. Pellets were diluted to $1 \times 106$ spermatozoa/mL in IVF medium to later coincubate with the oocytes. 
In vitro fertilization and embryo culture: Cumulus cells of mature COC's were removed with $0.1 \%$ hyaluronidase in IVM medium. Groups of 30 denuded oocytes were kept in $5 \% \mathrm{CO}_{2}$ and 39 ? for $30 \mathrm{~min}$, in $50 \mu \mathrm{L}$ drops of pre-equilibrated IVF medium, and $50 \mu \mathrm{L}$ of sperm suspension was added to each drop. The gametes were coincubated at 39 and 5\% CO2 for a period of 6h. After fertilization, group of 30 oocytes were, cultivated into $500 \mu \mathrm{L}$ of pre-equilibrated IVC medium at 39 and $5 \% \mathrm{CO}_{2}$. At $48 \mathrm{~h}$ after culture, the cleaved zygotes were evaluated under a stereomicroscope (Nikon SMZ 800, Japan).

\section{Statistical Analysis}

All variables were tested for normality using the ShapiroWilk test and homogeneity of variance was tested with the Levene test (group means). The determination of the differences among three evaluations per boar (carried out by the same analyst) was performed with the Friedman test for analysis of repeated measures in a randomized complete blocks (Boar) design. Values for MSI, PM, TM, MFI, NM and AI were grouped according to two groups of RCZ: Group1: $<23 \%$ and Group 2: $>23 \%$. Kruskal-
Wallis test was performed to detect differences between groups. The association between the RCZ with of independent variables was analysed as a Spearman correlation analysis. The values used for the correlation analysis and Kruskal-Wallis test were the averages of the three evaluations of each boar, understood as three values from a single physiological moment of the male. All statistical analyses were performed using R Program [14] version 3.4.2.

\section{Results}

The values of rate of cleaved zygotes fluctuated between 10 and 53.3\%. As can be seen in Figure 1, the boars that recorded an average less than $23 \%$ of cleaved zygotes (boars 1-5) had rates below this value in two of the three evaluations and presented at least one seminal quality variable below the threshold corresponding (NM higher than 70\%; AI higher than 75\%; MFI higher than 60\% and PM higher than 50\%) (Table 1). The average values of PM for boars 4 and 5 were very close to the threshold, therefore, the samples of these males were considered with low seminal quality.

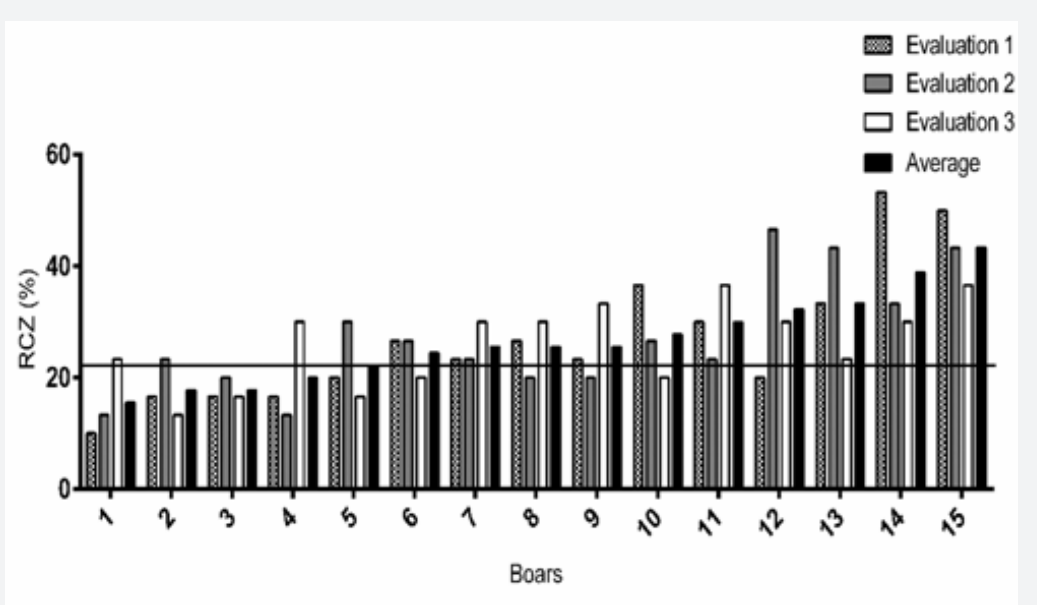

Figure 1: Rate of cleaved zygotes (RCZ) of 15 boars, evaluated every 7 days, 3 consecutive times.

Table 1: Averages of three consecutive analyzes of different seminal quality variables evaluated in refrigerated semen of 15 boars.

\begin{tabular}{|c|c|c|c|c|c|c|c|c|}
\hline \multirow{2}{*}{$\begin{array}{c}\text { Threshold } \\
\text { Boars }\end{array}$} & RCZ & MSI & PM & TM & MFI & NM & AI & $\begin{array}{c}\text { Samples with } \\
\text { Good Quality }\end{array}$ \\
\cline { 2 - 9 } & $\mathbf{2 3}$ & $\mathbf{7 0}$ & $\mathbf{5 0}$ & $\mathbf{6 0}$ & $\mathbf{6 0}$ & $\mathbf{7 0}$ & $\mathbf{7 5}$ & No \\
\hline 1 & 15.53 & 76 & 59.6 & 72.54 & 61.17 & 60.33 & 59 & 77.33 \\
\hline 2 & 17.73 & 81 & 43.13 & 60.14 & 56.69 & 52 & No \\
\hline 3 & 17.73 & 76 & 57.5 & 75.56 & 69.58 & 72 & No \\
\hline 4 & 19.97 & 76.33 & 50.18 & 67.18 & 87 & 94 & 83 & No \\
\hline 5 & 22.2 & 83.67 & 50.11 & 71.41 & 69.07 & 59.33 & 88.33 & No \\
\hline 6 & 24.4 & 87 & 56.01 & 74.3 & 86.08 & 80 & 84.33 & Yes \\
\hline 7 & 25.53 & 75.67 & 65.98 & 79.06 & 80.97 & 85 & 75.67 & Yes \\
\hline 8 & 25.53 & 81.33 & 72.58 & 86.85 & 79.41 & 89.67 & 85 & Yes \\
\hline 9 & 25.53 & 81 & 75.96 & 86.65 & 79.97 & 95 & 83.67 & Yes \\
\hline 10 & 27.73 & 82.33 & 63.73 & 77.24 & 77.21 & 92.67 & 87.33 & Yes \\
\hline 11 & 29.97 & 86 & 71.34 & 80.57 & 81.23 & 89 & 85 & Yes \\
\hline
\end{tabular}


Journal of Dairy \& Veterinary Sciences

\begin{tabular}{|l|c|c|c|c|c|c|c|c|}
\hline 12 & 32.2 & 79 & 64.64 & 76.09 & 83.37 & 89.33 & 79.33 & Yes \\
\hline 13 & 33.3 & 78 & 66.05 & 77.6 & 66.44 & 94.33 & 81.33 & Yes \\
\hline 14 & 38.87 & 72.33 & 52.55 & 64.69 & 78.67 & 91.33 & 83.33 & Yes \\
\hline 15 & 43.3 & 77.33 & 72.9 & 82.41 & 81.27 & 86.67 & 82.33 & Yes \\
\hline
\end{tabular}

RCZ: rate of cleaved zygotes; MSI: membrane structural integrity; PM: progressive motility; TM: total motility; MFI: membrane functional integrity; NM: normal morphology; Al: acrosomal integrity.

Likewise, the boars with an average greater than $23 \%$ of RCZ (boars 6-15) had at least two of the three evaluations with values up to $23 \%$ (Figure 1). In addition, all these boars had good seminal quality (Table 1). The average of RCZ in boars with less $23 \%$ (boars 1-5) of this variable was of $18.6 \%$, and in boars with a value greater than $23 \%$ (boars 6-15) was $30.6 \%$. Figure 2 shows differences between boars in the RCZ, and in the Friedman test no-significative differences $(P>0.05)$ within boars (three evaluations by boar carried out by the same analyst) were established for all variables (Table 2).

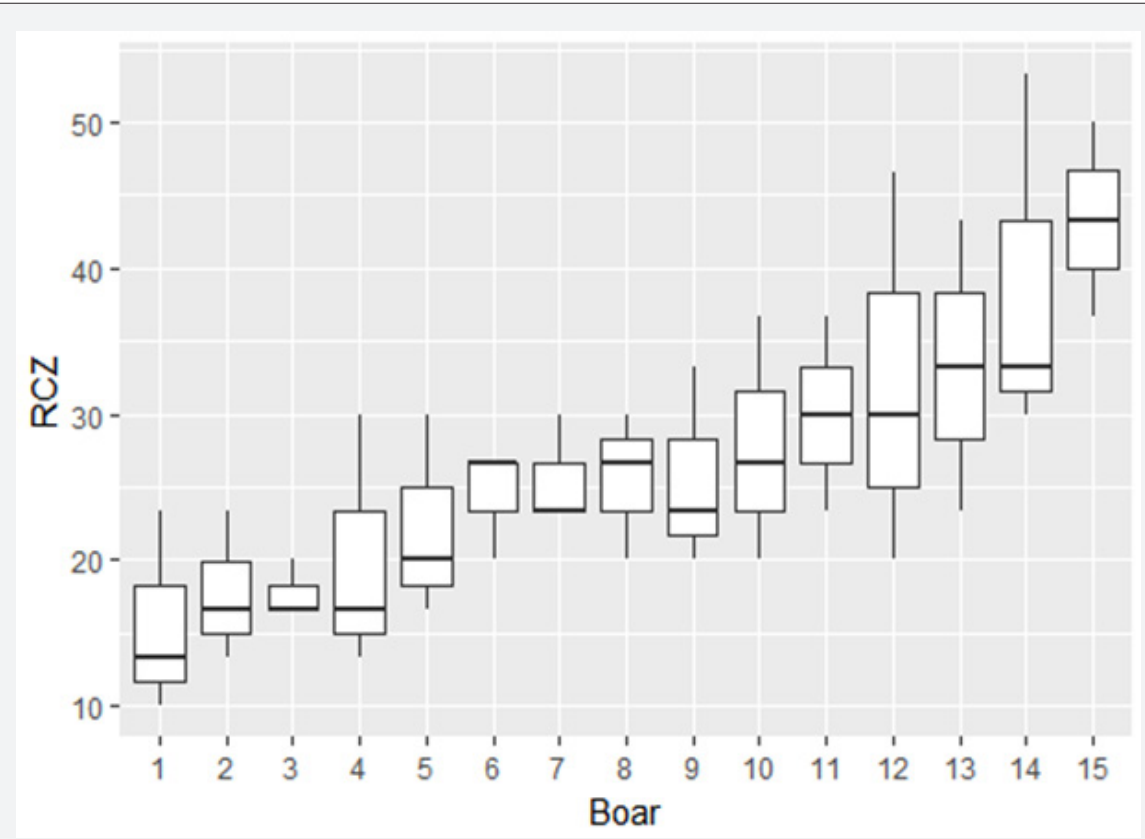

Figure 2: Boxplot of Rate of cleaved zygotes (RCZ) differences between boars evaluated three consecutive times, every 7 days.

Table 2: Differences within boars (three evaluations by boar) through the Friedman test for analysis of repeated measures in a randomized complete block design.

\begin{tabular}{|c|c|c|c|}
\hline Variable & Chi-squared & df & P \\
\hline RCZ & 0.2456 & 2 & 0.8844 \\
\hline MSI & 0.1017 & 2 & 0.9504 \\
\hline PM & 1.6 & 2 & 0.4493 \\
\hline TM & 3.6 & 2 & 0.1653 \\
\hline MFI & 0.9333 & 2 & 0.6271 \\
\hline NM & 2.3333 & 2 & 0.3114 \\
\hline AI & 4.5085 & 2 & 0.105 \\
\hline
\end{tabular}

RCZ: rate of cleaved zygotes; MSI: membrane structural integrity; PM: progressive motility; TM: total motility; MFI: membrane functional integrity; NM: normal morphology; Al: acrosomal integrity; $\mathrm{P}$ : probability value.
In the Kruskal-Wallis test were found significative differences $(\mathrm{P}<0.05)$ in PM, TM and NM, between group $1(<23 \% \mathrm{RCZ})$ and group 2 ( $>23 \% \mathrm{RCZ})$, and no significative differences $(\mathrm{P}>0.05)$ were evidenced for MSI, MFI and AI(Figure 3). In the association analysis (Spearman test) a high and significative correlation of RCZ with PM $(r=0.536, P=0.039)$ and NM $(r=0.547, P=0.034)$ was observed; the correlations between RCZ with MSI, AI, TM and MFI were no significative ( $\mathrm{P}>0.05$ ) (Table 3).

Table 3: Spearman correlation coefficients between rate of cleaved zygotes and other seminal quality variables.

\begin{tabular}{|c|c|c|c|c|c|c|c|}
\hline & & MSI & AI & PM & TM & NM & MFI \\
\hline \multirow[t]{2}{*}{ RCZ } & $\mathrm{r}$ & $0 . \overline{0}$ & 0.285 & 0.536 & 0.448 & 0.547 & 0.326 \\
\hline & $\mathrm{P}$ & 0.959 & 0.302 & 0.039 & 0.096 & 0.034 & 0.235 \\
\hline
\end{tabular}

RCZ: rate of cleaved zygotes; MSI: membrane structural integrity; $\mathrm{Al}$ : acrosomal integrity; PM: progressive motility; TM: total motility; NM: normal morphology; MFI: membrane functional integrity; r: correlation coefficient; P:probability value. 

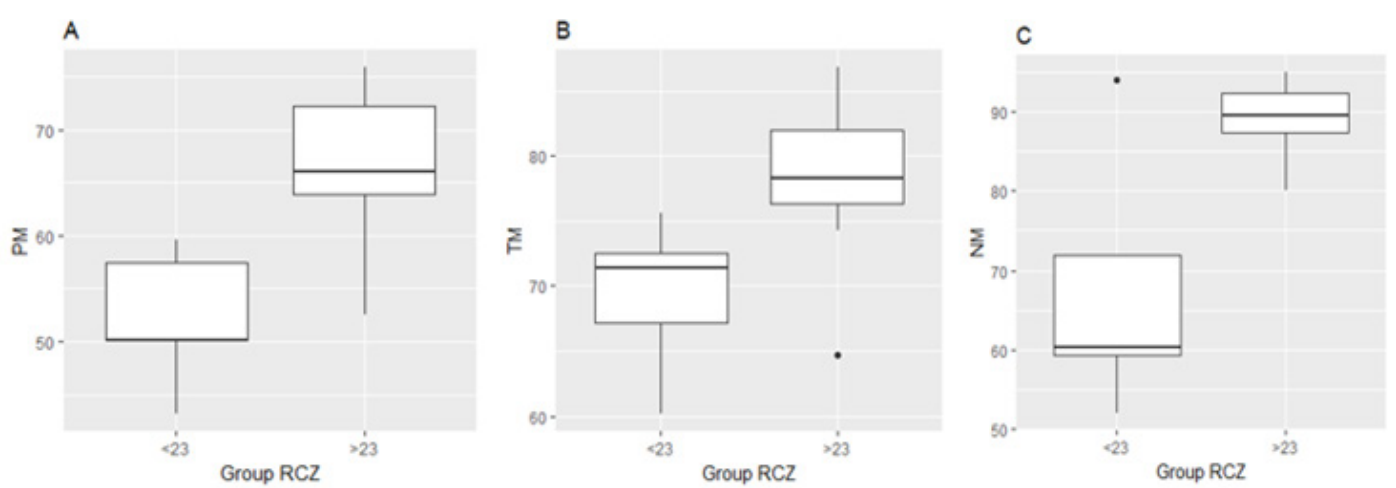

Figure 3: Boxplot of progressive motility (A), total motility (B) and normal morphology (C) differences in in two groups of rates of cleaved zygotes (RCZ).

\section{Discussion}

The RCZ obtained had values ranging from 10 to 53\%. Boars with an average less than $23 \%$ of RCZ had low seminal quality, and all boars with averages above this value had good seminal quality (Table 1). In this regard, it is reported that low seminal quality, specifically problems of motility, survival and morphology, is associated with boar reproductive disorders and low fertility [3]. Similarly, Gadea[2], reported that boar semen samples with high numbers of sperm abnormalities and cytoplasmic droplets, low MFI and AI correspond with low farrowing rate. In the study of Gil et al. [15], the average values of RCZ in boars with low and high freezability were $20.9 \%$ and 29.5\% respectively, and in the work of Abeydeera and Day [13] the RCZ obtained in a boar previously tested as high fertility was $40 \%$. In this work, the five boars of group 1 had an average of $18.6 \%$ of RCZ, and the ten boars of group 2 had $30.6 \%$, which coincides with the previous values. The findings discussed give relevant information to establish the RCZ as an important variable to evaluate the fertilizing ability and potential of embryo production of boar semen, and they permit to propose $23 \%$ as a possible threshold for to discriminate samples with low and high fertility.

Currently, there are very few studies where the RCZ is used to assess boar seminal quality, by cause of the test complexity and the difficulty of its standardization between laboratories, due to the high variability among the results between different IVF systems [11]. As shows in Figure 2, there were differences in RCZ values between the 15 evaluated boars, and there were no-significative differences $(\mathrm{P}>0.05)$ within the three consecutive evaluations of each boar (Table 2). This shows the consistency and repeatability of this tests and its capacity to discriminate between boars with different sperm fertility. These differences between boars in both in vivo and in vitro fertility have been shown in different studies [3]. Popwell and Flowers [16] postulated that one of the factors leading to erroneous conclusions is not to include the effect of the differences between ejaculates within the same boar in both IVF and in vivo fertility studies. In the design used in this study was possible to evaluate the differences within boar, and the average of the three boar evaluations was used after confirming that there were no differences.

After comparing each seminal quality variable grouped according to RCZ (group1: $<23 \%$ and group 2: $>23 \%$ ), normal morphology, total and progressive motility had significative differences $(\mathrm{P}<0.05)$, and their mean values in group 1 were lower than in group 2(Figure 3). Moreover, in the association analysis between RCZ with the other sperm characteristics, a high and significative correlation of RCZ with PM $(r=0.536, \mathrm{P}$ $=0.039$ ) was found. During fertilization, PM is a fundamental characteristic for the passage through the cumulosoophorus and ZP of the oocyte [1], however, the association of motility values with fertility is controversial, and it has been used to eliminate semen samples with apparent low quality [2]. In humans, there is evidence of a correlation between rate of in vitro fertilization and sperm motility evaluated through the CASA system [17], and in pigs, evaluated through the subjective visualization [18]. Likewise, correlation of the motility and the rate of penetration of immature oocytes has also been found[2]. However, in the study by Broekhuijse[11], there was no correlation of motility with RCZ, and according to the Popwell and Flowers [16] study, there was no association between motility and monospermic penetration rate of mature oocytes. It has been reported that very low values of motility can discriminate samples with low fertility[1].

In the present study PM values fluctuated between $28.7 \%$ and $83.6 \%$, and TM values, between $52.0 \%$ and $92.2 \%$, including samples with values extremely below the threshold for motility (equal to or greater than $60 \%$ of total motility and $50 \%$ of progressive motility). This situation may explain the differences in PM and TM values in groups with low and high RCZ (Figure 2 ), and the difference in the correlation found with motility compared to that reported by Broekhuijse[11] and Popwell and Flowers [16], who only used samples with values above the threshold for motility. It is important to note that although TM presents a questionable relationship with fertility, in agreement with the results found the PM has greater importance during fertilization. The threshold for TM used in this study (60\%) was 
taken from [1], and the threshold for PM was calculated in 49.4\%, based on the historical records of our laboratory (approximately $50 \%)$.

Normal morphology had high and significative correlation with RCZ $(r=0.547, \mathrm{P}=0.034)$ (Table 3$)$. There are antecedents with similar results using penetration rate of immature [2] and mature oocytes $[2,18]$. As evidenced in the sperm motility, in this study were incorporated samples with different percentages of normal morphology, ranging from $31 \%$ to $99 \%$. Within the sperm abnormalities that most affect the fertility of an ejaculate are the cytoplasmic droplets [2]. It is also considered that cytoplasmic droplets are the most frequent abnormality found in the semen of the boars used in artificial insemination centers, and therefore the major cause of discard of samples and boars; while abnormalities of head, mid piece and tail have a very low prevalence [19]. On the other hand, when performing artificial insemination with doses of $2 \times 109$ sperm/doses, the percentage of sperm with normal morphology explained a large part of the variance in litter size, which indicates that this variable is highly useful in the evaluation of seminal quality [18].

Non-significative correlations between RCZ with MSI, AI, TM and MFI were found ( $\mathrm{P}>0.05$ ) (Table 3 ). It is clear that variables such as MSI and TM, only indicate the viability of the sperm cell and not the capacity for capacitation, acrosomic reaction and fertilization [2]. With respect to MFI and AI are variables that provide relevant information on the biochemical functionality of the membrane, the acrosome membrane status, and therefore, the proper storage of the enzymes related to oocyte penetration. These tests could be combined to try to obtain greater predictive power of fertility in vivo and in vitro [6]. Other possible alternative is the creation of a multifactorial seminal quality index with other related seminal quality variables [20].

\section{Conclusion}

In conclusion, the results found in this research allow to establish the RCZ as an in vitro reference test because its consistence and repeatability to evaluate fertilizing capacity in commercial semen samples for those cases in which it is impossible to perform a field fertility evaluation with an enough number of sows, under strictly controlled conditions. This test provides information very useful in the in vitro seminal quality analysis, considering that when the average of the RCZ was less than $23 \%$, all boars had low seminal quality, and that all boars with an average greater than $23 \%$ had high seminal quality (acceptable value for all conventional in vitro variables). By the above, $23 \%$ is proposed as a possible threshold for RCZ.

\section{Acknowledgement}

The Pig farming Cooperative from the Coffee Triangle CERCAFE and the Topigenética Insemination Center for providing the seminal material to carry out the study.

\section{References}

1. Flowers WL (1997) Management of boars for efficient semen production. J Reprod Fertil Suppl 52: 67-78.
2. Gadea J, Matas C, Lucas X (1998) Prediction of porcine semen fertility by homologous in vitro penetration (hIVP) assay. Anim Reprod Sci 54(2): 95-108.

3. Ruiz-Sanchez AL, O'Donoghue R, Novak S, Dyck MK, Cosgrove JR, et al. (2006) The predictive value of routine semen evaluation and IVF technology for determining relative boar fertility. Theriogenology 66(4): 736-748.

4. Garner DL, Johnson LA (1995) Viability assessment of mammalian sperm using SYBR-14 and propidium iodide. Biol Reprod 53(2): 276284.

5. Perez-Llano B, Lorenzo JL, Yenes P, Trejo A, Garcia-Casado P (2001) A short hypoosmotic swelling test for the prediction of boar sperm fertility. Theriogenology 56(3): 387-398.

6. Perez-Llano B, Sala R, Reguera G, Garcia-Casado P (2009) Changes in subpopulations of boar sperm defined according to viability and plasma and acrosome membrane status observed during storage at 15 degrees C. Theriogenology 71(2): 311-317.

7. Lopez-Fernandez C, Perez-Llano B, Garcia-Casado P, Sala R, Gosalbez A, et al. (2008) Sperm DNA fragmentation in a random sample of the Spanish boar livestock. Anim Reprod Sci 103(1-2): 87-98.

8. Larsson B, Rodríguez-Martínez H (2000) Can we use in vitro fertilization tests to predict semen fertility? Anim Reprod Sci 60: 327-336.

9. Moce E, Graham JK (2008) In vitro evaluation of sperm quality. Anim Reprod Sci 105(1-2): 104-118.

10. Berger T, Anderson DL, Penedo MCT (1996) Porcine sperm fertilizing potential in relationship to sperm functional capacities. Anim Reprod Sci 44(4): 231-239.

11. Broekhuijse MLWJ (2012) Prediction of porcine male fertility. Doctoral Thesis. Netherlands Utrecht University, Utrecht, Europe.

12. Pursel VG, Johnson LA (1974) Glutaraldehyde fixation of boar spermatozoa for acrosome evaluation. Theriogenology 1(2): 63-68.

13. Abeydeera LR, Day BN (1997) Fertilization and subsequent development in vitro of pig oocytes inseminated in a modified trisbuffered medium with frozen-thawed ejaculated spermatozoa. Biol Reprod 57(4): 729-734.

14. R-Core-Team (2016) R: A language and environment for statistical computing. R Foundation for Statistical Computing, Vienna, Austria.

15. Gil MA, Roca J, Cremades T, Hernandez M, Vazquez JM, et al. (2005) Does multivariate analysis of post-thaw sperm characteristics accurately estimate in vitro fertility of boar individual ejaculates? Theriogenology 64(2): 305-316.

16. Popwell JM, Flowers WL (2004) Variability in relationships between semen quality and estimates of in vivo and in vitro fertility in boars. Anim Reprod Sci 81(1-2): 97-113.

17. Hirano Y, Shibahara H, Obara H, Suzuki T, Takamizawa S, et al. (2001) Relationships between sperm motility characteristics assessed by the computer-aided sperm analysis (CASA) and fertilization rates in vitro. J Assist Reprod Genet 18(4): 213-218.

18. Xu X, Pommier S, Arbov T, Hutchings B, Sotto W, Foxcroft GR (1998) In vitro maturation and fertilization techniques for assessment of semen quality and boar fertility. J Anim Sci 76(12): 3079-3089.

19. Gómez G, Mesa H, Sánches-Osorio J, Henao FJ (2015) Dynamics of Sus scrofa semen quality in Western-central region of Colombia. Bol Cient Mus His Nat 19: 139-53.

20. Díaz Franco O, Mesa H, Valencia Mejía JG, Gómez Londoño G, Henao Uribe FJ (2009) Evaluación de la integridad acrosomal y la funcionalidad bioquímica de la membrana espermática en cerdos reproductores con gotas citoplásmicas persistentes. Revista Científica FCV-LUZ 19: 500-505. 
This work is licensed under Creative Commons Attribution 4.0 License DOI: 10.19080/JDVS.2018.07.555719
Your next submission with Juniper Publishers will reach you the below assets

- Quality Editorial service

- Swift Peer Review

- Reprints availability

- E-prints Service

- Manuscript Podcast for convenient understanding

- Global attainment for your research

- Manuscript accessibility in different formats

( Pdf, E-pub, Full Text, Audio)

- Unceasing customer service

Track the below URL for one-step submission https://juniperpublishers.com/online-submission.php 